\title{
Second order asymptotic loss of the MLE of a truncation parameter for a truncated exponential family of distributions
}

\author{
M. Akahira and N. Ohyauchi \\ Institute of Mathematics, University of Tsukuba, Ibaraki, Japan
}

\begin{abstract}
For a truncated exponential family of distributions with a truncation parameter $\gamma$ and a natural parameter $\theta$ as a nuisance parameter, the stochastic expansions of bias-adjusted maximum likelihood estimators (MLEs) $\hat{\gamma}_{M L^{*}}^{\theta}$ and $\hat{\gamma}_{M L^{*}}$ of $\gamma$ when $\theta$ is known and when $\theta$ is unknown, respectively, are derived. The second order asymptotic loss of $\hat{\gamma}_{M L^{*}}$ relative to $\hat{\gamma}_{M L^{*}}^{\theta}$ is also obtained through their asymptotic variances. Further, some examples are given.
\end{abstract}

Keywords: Truncation parameter; Natural parameter; Maximum likelihood estimator; Asymptotic variance; Second order asymptotic loss; Pareto distribution

Mathematics Subject Classification: Primary 62F10; Secondary 62F12.

\section{Introduction}

In multiparameter cases, the estimation of an interest parameter has been discussed under suitable regularity conditions. It is important to grasp the effect on the presence of nuisance parameters in the estimation. In order to discriminate asymptotically efficient estimators, the concept of loss of information is useful (see Fisher (1925) and Rao (1961)). It is also known to be closely connected with the asymptotic deficiency discussed by Hodges and Lehmann (1970) (see Akahira $(1981,1986)$ ). On the other hand the conditional likelihood method is well known as a way of eliminating nuisance parameters (see, e.g. Basu (1977)). However, in the case when the regularity conditions are not necessarily satisfied, the asymptotic comparison of asymptotically efficient estimators has not been sufficiently discussed in the presence of nuisance parameters in higher order asymptotics yet.

For a truncated exponential family of distributions with a natural parameter $\theta$ and a truncation parameter $\gamma$ which is regarded as a typical non-regular case, Bar-Lev (1984) and Akahira (2013) 
considered a problem of estimating $\theta$ in the presence of $\gamma$ as a nuisance parameter. Let $\hat{\theta}_{M L}^{\gamma}$ and $\hat{\theta}_{M L}$ be the MLEs of $\theta$ based on a sample of size $n$ when $\gamma$ is known and when $\gamma$ is unknown, respectively. Let $\hat{\theta}_{M C L}$ be the maximum conditional likelihood estimator (MCLE). Then it was shown by BarLev (1984) that the MLEs $\hat{\theta}_{M L}^{\gamma}, \hat{\theta}_{M L}$ and the MCLE $\hat{\theta}_{M C L}$ have the same asymptotic normal distribution, hence they are shown to be asymptotically equivalent in the sense of having the same asymptotic variance. Further, Akahira (2013) compared them asymptotically up to the second order, i.e. the order $n^{-1}$, in the asymptotic variance, and showed that a bias-adjusted MLE $\hat{\theta}_{M L}^{*}$

and $\hat{\theta}_{M C L}$ were second order asymptotically equivalent, but they were asymptotically worse than $\hat{\theta}_{M L}^{\gamma}$ in the second order. The second order asymptotic losses on the asymptotic variance among them were also obtained.

In this paper we consider a problem of estimating $\gamma$ in the presence of $\theta$ as a nuisance parameter in exchanging an interest parameter for a nuisance parameter. Let $\hat{\gamma}_{M L}^{\theta}$ and $\hat{\gamma}_{M L}$ be the MLEs of $\gamma$ based on a sample of size $n$ when $\theta$ is known and when $\theta$ is unknown, respectively. The stochastic expansions of the bias-adjusted MLEs $\hat{\gamma}_{M L^{*}}^{\theta}$ and $\hat{\gamma}_{M L^{*}}$ are given, and the second order asymptotic loss of $\hat{\gamma}_{M L^{*}}$ relative to $\hat{\gamma}_{M L^{*}}^{\theta}$ is also obtained. Further some examples on the Pareto, truncated exponential and truncated normal cases are given.

\section{Truncated exponential family of distributions}

In a similar way to Bar-Lev (1984) and Akahira (2013), we consider the formulation as follows. Suppose that $X_{1}, X_{2}, \cdots, X_{n}, \cdots$ is a sequence of independent and identically distributed (i.i.d.) random variables according to $P_{\theta, \gamma}$, having a density

$$
f(x ; \theta, \gamma)= \begin{cases}\frac{a(x) e^{\theta u(x)}}{b(\theta, \gamma)} & \text { for } c<\gamma \leq x<d, \\ 0 & \text { otherwise }\end{cases}
$$

with respect to the Lebesgue measure, where $-\infty \leq c<d \leq \infty, a(\cdot)$ is a nonnegative and continuous almost surely, and $u(\cdot)$ is absolutely continuous with $d u(x) / d x \not \equiv 0$ over the interval $(\gamma, d)$. Let

$$
\Theta(\gamma):=\left\{\theta \mid 0<b(\theta, \gamma):=\int_{\gamma}^{d} a(x) e^{\theta u(x)} d x<\infty\right\}
$$

for $\gamma \in(c, d)$. Then it is shown that for any $\gamma_{1}, \gamma_{2} \in(c, d)$ with $\gamma_{1}<\gamma_{2}, \Theta\left(\gamma_{1}\right) \subset \Theta\left(\gamma_{2}\right)$. Assume that for any $\gamma \in(c, d), \Theta \equiv \Theta(\gamma)$ is a nonempty open interval. A family $\mathcal{P}:=\left\{P_{\theta, \gamma} \mid \theta \in \Theta, \gamma \in\right.$ $(c, d)\}$ of distributions $P_{\theta, \gamma}$ having a density (2.1) with a truncation parameter $\gamma$ and a natural 
parameter $\theta$ is called a truncated exponential family of distributions. Let

$$
\begin{aligned}
& k(\theta, \gamma):=a(\gamma) e^{\theta u(\gamma)} / b(\theta, \gamma), \\
& A(\theta, \gamma):=-\frac{1}{k^{2}(\theta, \gamma)}\left\{\frac{c_{\theta}(\gamma)}{a(\gamma)}+k(\theta, \gamma)\right\}
\end{aligned}
$$

with

$$
c_{\theta}(\gamma):=a^{\prime}(\gamma)+\theta a(\gamma) u^{\prime}(\gamma)
$$

Then

$$
\frac{1}{k(\theta, \gamma)}\left(\frac{\partial}{\partial \gamma} \log k(\theta, \gamma)\right)=\frac{c_{\theta}(\gamma)}{a(\gamma) k(\theta, \gamma)}+1=-k(\theta, \gamma) A(\theta, \gamma)
$$

Indeed, since, by $(2.2)$

$$
\frac{\partial b(\theta, \gamma)}{\partial \gamma}=-a(\gamma) e^{\theta u(\gamma)}
$$

it follows from (2.3), (2.4), (2.5) and (2.7) that

$$
\begin{aligned}
\frac{\partial}{\partial \gamma} \log k(\theta, \gamma) & =\frac{a^{\prime}(\gamma)}{a(\gamma)}+\theta u^{\prime}(\gamma)-\frac{1}{b(\theta, \gamma)}\left\{\frac{\partial}{\partial \gamma} b(\theta, \gamma)\right\} \\
& =\frac{a^{\prime}(\gamma)}{a(\gamma)}+\theta u^{\prime}(\gamma)+\frac{a(\gamma) e^{\theta u(\gamma)}}{b(\theta, \gamma)} \\
& =\frac{c_{\theta}(\gamma)}{a(\gamma)}+k(\theta, \gamma) \\
& =-k^{2}(\theta, \gamma) A(\theta, \gamma)
\end{aligned}
$$

hence (2.6) holds. In the subsequent sections we obtain the bias-adjusted MLE $\hat{\gamma}_{M L^{*}}^{\theta}$ and $\hat{\gamma}_{M L^{*}}$ of $\gamma$ for known and unknown $\theta$, respectively. Calculating their asymptotic variances based on their stochastic expansions, we get the second order asymptotic loss of $\hat{\gamma}_{M L^{*}}$ relative to $\hat{\gamma}_{M L^{*}}^{\theta}$. Some examples are given, and the proofs of theorems are located in appendix. 


\section{The bias-adjusted MLE $\hat{\gamma}_{M L^{*}}^{\theta}$ of $\gamma$ when $\theta$ is known}

For given $\boldsymbol{x}:=\left(x_{1}, \cdots, x_{n}\right)$ satisfying $\gamma<x_{(1)}:=\min _{1 \leq i \leq n} x_{i}$ and $x_{(n)}:=\max _{1 \leq i \leq n} x_{i}<d$, the likelihood function of $\gamma$ is given by

$$
L^{\theta}(\gamma ; \boldsymbol{x})=\frac{1}{b^{n}(\theta, \gamma)}\left\{\prod_{i=1}^{n} a\left(x_{i}\right)\right\} \exp \left\{\theta \sum_{i=1}^{n} u\left(x_{i}\right)\right\}
$$

when $\theta$ is known. From (2.2) and (3.1) it follows that the MLE $\hat{\gamma}_{M L}^{\theta}$ of $\gamma$ is given by $X_{(1)}:=$ $\min _{1 \leq i \leq n} X_{i}$. Let $T_{(1)}:=n\left(X_{(1)}-\gamma\right)$. Then we have the following.

Theorem 3.1. For the truncated exponential family $\mathcal{P}$ of distributions having a density (2.1) with a truncation parameter $\gamma$ and a natural parameter $\theta$, let $\hat{\gamma}_{M L^{*}}^{\theta}=X_{(1)}^{*}$ be a bias-adjusted $M L E$ of $\gamma$ such that

$$
X_{(1)}^{*}:=X_{(1)}-\frac{1}{\hat{k}_{\theta} n}
$$

where $\hat{k}_{\theta}=k\left(\theta, X_{(1)}\right)$. Then the stochastic expansion of $T_{(1)}^{*}:=n\left(X_{(1)}^{*}-\gamma\right)$ is given by

$$
T_{(1)}^{*}=T_{(1)}-\frac{1}{k}+\frac{1}{k n}\left(\frac{\partial}{\partial \gamma} \log k\right) T_{(1)}+O_{p}\left(\frac{1}{n^{2}}\right)
$$

where $k=k(\theta, \gamma)$, and the second order asymptotic mean and variance are given by

$$
\begin{aligned}
& E_{\gamma}\left[T_{(1)}^{*}\right]=O\left(\frac{1}{n^{2}}\right) \\
& V_{\gamma}\left(k T_{(1)}^{*}\right)=1-\frac{2}{k n}\left(\frac{\partial}{\partial \gamma} \log k\right)+O\left(\frac{1}{n^{2}}\right),
\end{aligned}
$$

respectively.

\section{The bias-adjusted MLE $\hat{\gamma}_{M L^{*}}$ of $\gamma$ when $\theta$ is unknown}

For any $\gamma \in(c, d), \log b(\theta, \gamma)$ is strictly convex and infinitely differentiable in $\theta \in \Theta$ and

$$
\lambda_{j}(\theta, \gamma):=\frac{\partial^{j}}{\partial \theta^{j}} \log b(\theta, \gamma)
$$


is the $j$-th cumulant corresponding to $(2.1)$ for $j=1,2, \cdots$. For given $\boldsymbol{x}$ satisfying $\gamma<x_{(1)}$ and $x_{(n)}<d$, the likelihood function of $\gamma$ and $\theta$ is given by

$$
L(\gamma, \theta ; \boldsymbol{x})=\frac{1}{b^{n}(\theta, \gamma)}\left\{\prod_{i=1}^{n} a\left(x_{i}\right)\right\} \exp \left\{\theta \sum_{i=1}^{n} u\left(x_{i}\right)\right\} .
$$

Let $\hat{\gamma}_{M L}$ and $\hat{\theta}_{M L}$ be the MLEs of $\gamma$ and $\theta$, respectively. From (4.2) it is seen that $\hat{\gamma}_{M L}=X_{(1)}$ and $L\left(X_{(1)}, \hat{\theta}_{M L} ; \boldsymbol{X}\right)=\sup _{\theta \in \Theta} L\left(X_{(1)}, \theta ; \boldsymbol{X}\right)$, hence $\hat{\theta}_{M L}$ satisfies the likelihood equation

$$
\frac{1}{n} \sum_{i=1}^{n} u\left(X_{i}\right)-\lambda_{1}\left(\hat{\theta}_{M L}, X_{(1)}\right)=0
$$

where $\boldsymbol{X}=\left(X_{1}, \cdots, X_{n}\right)$. Let $\lambda_{2}=\lambda_{2}(\theta, \gamma)$ and $\hat{U}=\sqrt{\lambda_{2} n}\left(\hat{\theta}_{M L}-\theta\right)$. Then we have the following.

Theorem 4.1. For the truncated exponential family $\mathcal{P}$ of distributions having a density (2.1) with a truncation parameter $\gamma$ and a natural parameter $\theta$, let $\hat{\gamma}_{M L^{*}}=X_{(1)}^{* *}$ be a bias-adjusted $M L E$ of $\gamma$ such that

$$
\begin{aligned}
X_{(1)}^{* *}:=X_{(1)} & -\frac{1}{\hat{k} n}+\frac{1}{\hat{k}^{2} \hat{\lambda}_{2} n^{2}}\left(\frac{\partial \hat{k}}{\partial \theta}\right)\left\{\frac{1}{\hat{k}}\left(\frac{\partial \hat{\lambda}_{1}}{\partial \gamma}\right)+\frac{\hat{\lambda}_{3}}{2 \hat{\lambda}_{2}}\right\} \\
& -\frac{1}{2 \hat{k}^{2} \hat{\lambda}_{2} n^{2}}\left\{\frac{\partial^{2} \hat{k}}{\partial \theta^{2}}-\frac{2}{\hat{k}}\left(\frac{\partial \hat{k}}{\partial \theta}\right)^{2}\right\},
\end{aligned}
$$

where $\hat{k}=k\left(\hat{\theta}_{M L}, X_{(1)}\right), \partial^{j} \hat{k} / \partial \theta^{j}=\left(\partial^{j} k / \partial \theta^{j}\right)\left(\hat{\theta}_{M L}, X_{(1)}\right)(j=1,2), \hat{\lambda}_{j}=\lambda_{j}\left(\hat{\theta}_{M L}, X_{(1)}\right)(j=2,3)$ and $\partial \hat{\lambda}_{1} / \partial \gamma=\left(\partial \lambda_{1} / \partial \gamma\right)\left(\hat{\theta}_{M L}, X_{(1)}\right)$. Then the stochastic expansion of $T_{(1)}^{* *}:=n\left(X_{(1)}^{* *}-\gamma\right)$ is given by

$$
\begin{array}{r}
T_{(1)}^{* *}=T_{(1)}-\frac{1}{k}+\frac{1}{k^{2} \sqrt{\lambda_{2} n}}\left(\frac{\partial k}{\partial \theta}\right)\left\{\hat{U}+\frac{1}{\sqrt{\lambda_{2} n}}\left(\frac{1}{k}\left(\frac{\partial \lambda_{1}}{\partial \gamma}\right)+\frac{\lambda_{3}}{2 \lambda_{2}}\right)\right\} \\
+\frac{1}{k n}\left(\frac{\partial}{\partial \gamma} \log k\right) T_{(1)}+\frac{1}{2 k^{2} \lambda_{2} n}\left\{\frac{\partial^{2} k}{\partial \theta^{2}}-\frac{2}{k}\left(\frac{\partial k}{\partial \theta}\right)^{2}\right\}\left(\hat{U}^{2}-1\right) \\
+O_{p}\left(\frac{1}{n \sqrt{n}}\right),
\end{array}
$$

where $k=k(\theta, \gamma), \lambda_{j}=\lambda_{j}(\theta, \gamma)(j=1,2,3)$, and the second order asymptotic mean and variance are given by

$$
E_{\theta, \gamma}\left[T_{(1)}^{* *}\right]=O\left(\frac{1}{n \sqrt{n}}\right),
$$




$$
V_{\theta, \gamma}\left(k T_{(1)}^{* *}\right)=1-\frac{2}{k n}\left(\frac{\partial}{\partial \gamma} \log k\right)+\frac{1}{\lambda_{2} n}\left(u(\gamma)-\lambda_{1}\right)^{2}+O\left(\frac{1}{n \sqrt{n}}\right) .
$$

\section{The second order asymptotic loss of $\hat{\gamma}_{M L^{*}}$ relative to $\hat{\gamma}_{M L^{*}}^{\theta}$}

From the results in previous sections, we can asymptotically compare the bias-adjusted MLEs $\hat{\gamma}_{M L^{*}}$ and $\hat{\gamma}_{M L^{*}}$ of $\gamma$ using their second order asymptotic variances as follows.

Theorem 5.1. For the truncated exponential family $\mathcal{P}$ of distributions having a density (2.1) with a truncation parameter $\gamma$ and a natural parameter $\theta$, let $\hat{\gamma}_{M L^{*}}$ and $\hat{\gamma}_{M L^{*}}$ be the bias-adjusted MLEs of $\gamma$ when $\theta$ is known and when $\theta$ is unknown, respectively. Then the second order asymptotic loss of $\hat{\gamma}_{M L^{*}}=X_{(1)}^{* *}$ relative to $\hat{\gamma}_{M L^{*}}^{\theta}=X_{(1)}^{*}$ is given by

$$
d_{n}\left(\hat{\gamma}_{M L^{*}}, \hat{\gamma}_{M L^{*}}^{\theta}\right):=n\left\{V_{\theta, \gamma}\left(k T_{(1)}^{* *}\right)-V_{\gamma}\left(k T_{(1)}^{*}\right)\right\}=\frac{\left\{u(\gamma)-\lambda_{1}\right\}^{2}}{\lambda_{2}}+o(1)
$$

as $n \rightarrow \infty$.

The proof is straightforward from Theorems 3.1 and 4.1 .

Remark 5.1. The second order asymptotic loss of $\hat{\gamma}_{M L^{*}}$ relative to $\hat{\gamma}_{M L^{*}}^{\theta}$ coincides with that of the bias-adjusted MLE $\hat{\theta}_{M L}^{*}$ of $\theta$ when $\gamma$ is unknown relative to the MLE $\hat{\theta}_{M L}^{\gamma}$ of $\theta$ when $\gamma$ is known, which seems to show a dual relation on the second order asymptotic loss (see Akahira (2013)). It is noted that the standardization is necessary in the comparison.

Remark 5.2. Suppose that $X_{1}, X_{2}, \cdots, X_{n}, \cdots$ is a sequence of i.i.d. random variables according to an upper-truncated exponential family $\mathcal{P}^{\prime}$ of distributions with a density

$$
f(x ; \theta, \nu)= \begin{cases}\frac{a(x) e^{\theta u(x)}}{b(\theta, \nu)} & \text { for } c<x \leq \nu<d \\ 0 & \text { otherwise }\end{cases}
$$

with respect to the Lebesgue measure, where $b(\theta, \nu)$ is a normalizing factor. Letting $Y_{i}=-X_{i}$ $(i=1,2, \cdots)$, and returning to the case of the lower-truncated exponential family with (2.1), we may obtain similar results to the above in a problem of estimating an upper truncation parameter $\nu$ in the presence of $\theta$ as a nuisance parameter. 


\section{Examples}

Some examples on the second order asymptotic loss of the estimators are given for the Pareto distribution, a truncated exponential distribution and a truncated normal distribution. Note that the examples are treated in Akahira (2013).

Example 6.1 (Pareto distribution). Let $c=0, d=\infty, a(x)=1 / x$ and $u(x)=-\log x$ for $0<\gamma \leq x<\infty$ in the density (2.1). Then $b(\theta, \gamma)=1 /\left(\theta \gamma^{\theta}\right)$ for $\theta \in \Theta=(0, \infty)$, and it follows from (2.2) and (2.3) that $k(\theta, \gamma)=\theta / \gamma, \partial k / \partial \theta=1 / \gamma$ and $\partial k / \partial \gamma=-\theta / \gamma^{2}$. When $\theta$ is known, it follows from (3.2) that the bias-adjusted MLE $\hat{\gamma}_{M L^{*}}^{\theta}$ of $\gamma$ is given by

$$
X_{(1)}^{*}=\left(1-\frac{1}{\theta n}\right) X_{(1)}
$$

hence by (3.4) and (3.5)

$$
\begin{aligned}
& E_{\gamma}\left[T_{(1)}^{*}\right]=O\left(\frac{1}{n^{2}}\right), \\
& V_{\gamma}\left(\frac{\theta}{\gamma} T_{(1)}^{*}\right)=1+\frac{2}{n \theta}+O\left(\frac{1}{n^{2}}\right),
\end{aligned}
$$

as $n \rightarrow \infty$, where $T_{(1)}^{*}=n\left(X_{(1)}^{*}-\gamma\right)$. On the other hand, in the Pareto case, it is known that the uniformly minimum variance unbiased (UMVU) estimator of $\gamma$ is given by

$$
\hat{\gamma}_{U M V U}^{\theta}:=\left(1-\frac{1}{n \theta}\right) X_{(1)}=X_{(1)}^{*}
$$

and its variance is

$$
V_{\gamma}\left(\hat{\gamma}_{U M V U}^{\theta}\right)=\frac{\gamma^{2}}{n \theta(n \theta-2)}
$$

(see, e.g. Voinov and Nikulin (1993)), hence

$$
V_{\gamma}\left(\frac{n \theta}{\gamma} \hat{\gamma}_{U M V U}^{\theta}\right)=\frac{n \theta}{n \theta-2}=1+\frac{2}{n \theta}+O\left(\frac{1}{n^{2}}\right)
$$

which is equal to (6.1) up to the order $1 / n$ as $n \rightarrow \infty$.

Next we consider the case when $\theta$ is unknown. Since $\partial^{2} k / \partial \theta^{2}=0, \lambda_{1}=-(1 / \theta)-\log \gamma$, $\lambda_{2}=1 / \theta^{2}, \lambda_{3}=-2 / \theta^{3}$ and $\partial \lambda_{1} / \partial \gamma=-1 / \gamma$, it follows from (4.4) that the bias-adjusted MLE $\hat{\gamma}_{M L^{*}}$ 
of $\gamma$ is given by

$$
X_{(1)}^{* *}=\left\{1-\left(\frac{1}{n}+\frac{1}{n^{2}}\right) \frac{1}{\hat{\theta}_{M L}}\right\} X_{(1)}
$$

where $\hat{\theta}_{M L}=n / \sum_{i=1}^{n} \log \left(X_{(i)} / X_{(1)}\right)$ from (4.3). Since $(\partial / \partial \gamma) \log k=-1 / \gamma$, we have from (4.6) and (4.7)

$$
\begin{aligned}
& E_{\theta, \gamma}\left[T_{(1)}^{* *}\right]=O\left(\frac{1}{n \sqrt{n}}\right) \\
& V_{\theta, \gamma}\left(\frac{\theta}{\gamma} T_{(1)}^{* *}\right)=1+\frac{1}{n}\left(1+\frac{2}{\theta}\right)+O\left(\frac{1}{n^{2}}\right)
\end{aligned}
$$

as $n \rightarrow \infty$, where $T_{(1)}^{* *}=n\left(X_{(1)}^{* *}-\gamma\right)$. On the other hand, in the Pareto case, it is known that the UMVU estimator of $\gamma$ is given by

$$
\hat{\gamma}_{U M V U}=X_{(1)}-\frac{X_{(1)}}{(n-1) \hat{\theta}_{M L}}
$$

and its variance is

$$
V_{\theta, \gamma}\left(\hat{\gamma}_{U M V U}\right)=\frac{\gamma^{2}}{(n-1) \theta(n \theta-2)}
$$

(see, e.g. Voinov and Nikulin (1993)), hence

$$
V_{\theta, \gamma}\left(\frac{n \theta}{\gamma} \hat{\gamma}_{U M V U}\right)=\frac{n^{2} \theta}{(n-1)(n \theta-2)}=1+\frac{1}{n}\left(1+\frac{2}{\theta}\right)+O\left(\frac{1}{n^{2}}\right),
$$

which is equal to (6.3) up to the order $1 / n$ as $n \rightarrow \infty$. It also follows from (5.1), (6.1) and (6.3) that the second order asymptotic loss of $\hat{\gamma}_{M L^{*}}=X_{(1)}^{* *}$ relative to $\hat{\gamma}_{M L^{*}}^{\theta}=X_{(1)}^{*}$ is given by

$$
d_{n}\left(\hat{\gamma}_{M L^{*}}, \hat{\gamma}_{M L^{*}}^{\theta}\right)=1+o(1)
$$

as $n \rightarrow \infty$. From (6.2) and (6.4) it follows that the second order asymptotic loss of $\hat{\gamma}_{U M V U}$ relative to $\hat{\gamma}_{U M V U}^{\theta}$ is

$$
\begin{aligned}
d\left(\hat{\gamma}_{U M V U}, \hat{\gamma}_{U M V U}^{\theta}\right): & =n\left\{V_{\theta, \gamma}\left(\frac{n \theta}{\gamma} \hat{\gamma}_{U M V U}\right)-V_{\gamma}\left(\frac{n \theta}{\gamma} \hat{\gamma}_{U M V U}^{\theta}\right)\right\} \\
& =\frac{n^{2} \theta}{(n-1)(n \theta-2)}=1+O\left(\frac{1}{n}\right),
\end{aligned}
$$

which coincides with (6.5) as $n \rightarrow \infty$. 
Example 6.2 (Truncated exponential distribution). Let $c=-\infty, d=\infty, a(x) \equiv 1$ and $u(x)=-x$ for $-\infty<\gamma \leq x<\infty$ in the density (2.1). Since $b(\theta, \gamma)=e^{-\theta \gamma} / \theta$ for $\theta \in \Theta=(0, \infty)$, it follows from (4.1) that $\lambda_{1}(\theta, \gamma)=-\gamma-(1 / \theta), \lambda_{2}(\theta, \gamma)=1 / \theta^{2}, \lambda_{3}(\theta, \gamma)=-2 / \theta^{3}$. Since, by $(2.3), k(\theta, \gamma)=\theta$, it is seen that $(\partial / \partial \theta) k(\theta, \gamma)=1,\left(\partial^{2} / \partial \theta^{2}\right) k(\theta, \gamma)=0$. When $\theta$ is known, it follows from (3.2) that the bias-adjusted MLE $\hat{\gamma}_{M L^{*}}^{\theta}$ of $\gamma$ is given by

$$
X_{(1)}^{*}=X_{(1)}-\frac{1}{n \theta}
$$

When $\theta$ is unknown, it is seen from (4.3) that the MLE $\hat{\theta}_{M L}$ of $\theta$ is given by $\hat{\theta}_{M L}=1 /\left(\bar{X}-X_{(1)}\right)$, hence by (4.4) the bias-adjusted MLE $\hat{\gamma}_{M L^{*}}$ of $\gamma$ is given by

$$
X_{(1)}^{* *}=X_{(1)}-\left(\frac{1}{n}+\frac{1}{n^{2}}\right)\left(\bar{X}-X_{(1)}\right)
$$

From Theorem 5.1 it follows that the second order asymptotic loss of $\hat{\gamma}_{M L^{*}}=X_{(1)}^{* *}$ for unknown $\theta$ relative to $\hat{\gamma}_{M L^{*}}^{\theta}=X_{(1)}^{*}$ for known $\theta$ is given by

$$
d_{n}\left(\hat{\gamma}_{M L^{*}}, \hat{\gamma}_{M L^{*}}^{\theta}\right)=1+o(1)
$$

as $n \rightarrow \infty$.

Example 6.3 (Truncated normal distribution). Let $c=-\infty, d=\infty, a(x)=e^{-x^{2} / 2}$ and $u(x)=x$ for $-\infty<\gamma \leq x<\infty$ in the density (2.1). Since $b(\theta, \gamma)=\Phi(\theta-\gamma) / \phi(\theta)$ for $\theta \in \Theta=(-\infty, \infty)$, it follows from (4.1) that

$$
\begin{aligned}
& \lambda_{1}(\theta, \gamma)=\theta+\rho(\theta-\gamma), \quad \frac{\partial \lambda_{1}(\theta, \gamma)}{\partial \gamma}=(\theta-\gamma) \rho(\theta-\gamma)+\rho^{2}(\theta-\gamma), \\
& \lambda_{2}(\theta, \gamma)=1-(\theta-\gamma) \rho(\theta-\gamma)-\rho^{2}(\theta-\gamma), \\
& \lambda_{3}(\theta, \gamma)=\rho(\theta-\gamma)\left\{2 \rho^{2}(\theta-\gamma)+3(\theta-\gamma) \rho(\theta-\gamma)+(\theta-\gamma)^{2}-1\right\},
\end{aligned}
$$

where $\rho(t):=\phi(t) / \Phi(t)$ with

$$
\Phi(x)=\int_{-\infty}^{x} \phi(t) d t, \quad \phi(t)=\frac{1}{\sqrt{2 \pi}} e^{-t^{2} / 2} \text { for }-\infty<t<\infty .
$$

We also have from $(2.3)$

$$
k(\theta, \gamma)=\rho(\theta-\gamma), \quad \frac{\partial k(\theta, \gamma)}{\partial \theta}=-(\theta-\gamma) \rho(\theta-\gamma)-\rho^{2}(\theta-\gamma)
$$




$$
\begin{aligned}
& \frac{\partial^{2} k(\theta, \gamma)}{\partial \theta^{2}}=\rho(\theta-\gamma)\left\{2 \rho^{2}(\theta-\gamma)+3(\theta-\gamma) \rho(\theta-\gamma)+(\theta-\gamma)^{2}-1\right\} \\
& \frac{\partial k(\theta, \gamma)}{\partial \gamma}=(\theta-\gamma) \rho(\theta-\gamma)+\rho^{2}(\theta-\gamma)
\end{aligned}
$$

When $\theta$ is known, it follows from (3.2) that the bias-adjusted MLE $\hat{\gamma}_{M L^{*}}^{\theta}$ of $\gamma$ is

$$
X_{(1)}^{*}=X_{(1)}-\frac{1}{\rho\left(\theta-X_{(1)}\right) n} .
$$

When $\theta$ is unknown, it is seen from (4.3) that the MLE $\hat{\theta}_{M L}$ of $\theta$ satisfies the equation

$$
\rho\left(\hat{\theta}_{M L}-X_{(1)}\right)=\bar{X}-\hat{\theta}_{M L}
$$

hence the bias-adjusted MLE $\hat{\gamma}_{M L^{*}}$ of $\gamma$ is

$$
X_{(1)}^{* *}=X_{(1)}-\frac{1}{n\left(\bar{X}-\hat{\theta}_{M L}\right)}+\frac{1-\left(\bar{X}-X_{(1)}\right)\left(\bar{X}-X_{(1)}+\bar{X}-\hat{\theta}_{M L}\right)}{2 n^{2}\left(\bar{X}-\hat{\theta}_{M L}\right)\left\{1-\left(\bar{X}-\hat{\theta}_{M L}\right)\left(\bar{X}-X_{(1)}\right)\right\}} .
$$

From Theorem 5.1 it follows that the second order asymptotic loss of $X_{(1)}^{* *}$ for unknown $\theta$ relative to $X_{(1)}^{*}$ for known $\theta$ is given by

$$
d_{n}\left(X_{(1)}^{* *}, X_{(1)}^{*}\right)=\frac{\{\theta-\gamma+\rho(\theta-\gamma)\}^{2}}{1-(\theta-\gamma) \rho(\theta-\gamma)-\rho^{2}(\theta-\gamma)}+o(1)
$$

as $n \rightarrow \infty$.

\section{Concluding Remarks}

In a truncated exponential family of distributions with a two-dimensional parameter $(\theta, \gamma)$, we considered the estimation problem of a truncation parameter $\gamma$ in the presence of a natural parameter $\theta$ as a nuisance parameter. Using the stochastic expansions of the bias-adjusted MLEs $\hat{\gamma}_{M L^{*}}^{\theta}$ and $\hat{\gamma}_{M L^{*}}$ of $\gamma$ when $\theta$ is known and when $\theta$ is unknown, respectively, we obtained their second order asymptotic variances, from which the second order asymptotic loss of $\hat{\gamma}_{M L^{*}}$ relative to $\hat{\gamma}_{M L^{*}}^{\theta}$ was derived. As is stated in Remark 5.1, the second order asymptotic loss coincides with that of the bias-adjusted MLE $\hat{\theta}^{*}$ of $\theta$ when $\gamma$ is unknown relative to the MLE $\hat{\theta}_{M L}^{\gamma}$ of $\theta$ when $\gamma$ is known, which means that the invariance on the second order asymptotic loss holds even if the exchange of an interest parameter for a nuisance parameter is done.

The results of Theorems 3.1, 4.1 and 5.1 can be extended to the case of a two-sided truncated 
exponential family of distributions with two truncation parameters $\gamma$ and $\nu$ and a natural parameter $\theta$ as a nuisance parameter, including an upper-truncated Pareto distribution which is important in applications (see Akahira and Ohyauchi (2015)). For such a family of distributions, Akahira et.al (2014) compared a bias-adjusted MLE $\hat{\theta}_{M L}^{*}$ and MCLE $\hat{\theta}_{M C L}$ of $\theta$ for unknown $\gamma$ and $\nu$ as nuisance parameters with the MLE $\hat{\theta}_{M L}^{\gamma, \nu}$ of $\theta$ for known $\gamma$ and $\nu$, and obtained the second order asymptotic losses of $\hat{\theta}_{M L}^{*}$ and $\hat{\theta}_{M C L}$ relative to $\hat{\theta}_{M L}^{\gamma, \nu}$.

\section{Appendix}

Before proving Theorems 3.1 and 4.1, we prepare two lemmas.

Lemma A.1. The second order asymptotic density of $T_{(1)}$ is given by

$$
\begin{aligned}
& f_{T_{(1)}}(t)=k(\theta, \gamma) e^{-k(\theta, \gamma) t}+ \frac{k(\theta, \gamma)}{a(\gamma) b(\theta, \gamma) n}\left\{c_{\theta}(\gamma) b(\theta, \gamma)+a^{2}(\gamma) e^{\theta u(\gamma)}\right\} \\
& \cdot\left\{t-\frac{1}{2} k(\theta, \gamma) t^{2}\right\} e^{-k(\theta, \gamma) t}+O\left(\frac{1}{n^{2}}\right)
\end{aligned}
$$

for $t>0$, and

$$
\begin{aligned}
& E_{\theta, \gamma}\left(T_{(1)}\right)=\frac{1}{k(\theta, \gamma)}+\frac{1}{n} A(\theta, \gamma)+O\left(\frac{1}{n^{2}}\right), \\
& E_{\theta, \gamma}\left(T_{(1)}^{2}\right)=\frac{2}{k^{2}(\theta, \gamma)}-\frac{6\left\{c_{\theta}(\gamma) b(\theta, \gamma)+a^{2}(\gamma) e^{\theta u(\gamma)}\right\}}{a(\gamma) b(\theta, \gamma) k^{3}(\theta, \gamma) n}+O\left(\frac{1}{n^{2}}\right),
\end{aligned}
$$

where $k(\theta, \gamma), A(\theta, \gamma)$ and $c_{\theta}(\gamma)$ are given as (2.3), (2.4) and (2.5), respectively.

The proof of (A.1) is omitted, since it is given in Akahira (2013), and (A.2) and (A.3) are straightforwardly obtained.

Lemma A.2. Let $\hat{U}:=\sqrt{\lambda_{2}(\theta, \gamma) n}\left(\hat{\theta}_{M L}-\theta\right)$. Then the asymptotic expectation of $\hat{U}, \hat{U}^{2}$ and $\hat{U} T_{(1)}$ are given by

$$
\begin{aligned}
& E_{\theta, \gamma}(\hat{U})=-\frac{1}{\sqrt{\lambda_{2} n}}\left\{\frac{1}{k}\left(\frac{\partial \lambda_{1}}{\partial \gamma}\right)+\frac{\lambda_{3}}{2 \lambda_{2}}\right\}+O\left(\frac{1}{n \sqrt{n}}\right), \\
& E_{\theta, \gamma}\left(\hat{U}^{2}\right)=1+O\left(\frac{1}{n}\right), \\
& E_{\theta, \gamma}\left(\hat{U} T_{(1)}\right)=\frac{1}{k \sqrt{\lambda_{2} n}}\left\{u(\gamma)-\lambda_{1}-\frac{\lambda_{3}}{2 \lambda_{2}}\right\}+O\left(\frac{1}{n \sqrt{n}}\right),
\end{aligned}
$$

where $\lambda_{j}=\lambda_{j}(\theta, \gamma)(j=1,2,3)$ and $k=k(\theta, \gamma)$. 
Proof The formulae (A.4) and (A.5) are given in Akahira (2013). Letting

$$
Z_{1}:=\frac{1}{\sqrt{\lambda_{2} n}} \sum_{i=1}^{n}\left\{u\left(X_{i}\right)-\lambda_{1}\right\}
$$

we have

$$
\hat{U}=Z_{1}-\frac{\lambda_{3}}{2 \lambda_{2}^{3 / 2} \sqrt{n}} Z_{1}^{2}-\frac{1}{\sqrt{\lambda_{2} n}}\left(\frac{\partial \lambda_{1}}{\partial \gamma}\right) T_{(1)}+O_{p}\left(\frac{1}{n}\right)
$$

(see Theorem 2 in Akahira (2013)). Since

$$
\begin{aligned}
& E_{\theta, \gamma}\left(Z_{1} T_{(1)}\right)=\frac{1}{k \sqrt{\lambda_{2} n}}\left\{u(\gamma)-\lambda_{1}+\frac{2}{k}\left(\frac{\partial \lambda_{1}}{\partial \gamma}\right)\right\}+O\left(\frac{1}{n \sqrt{n}}\right) \\
& E_{\theta, \gamma}\left(Z_{1}^{2} T_{(1)}\right)=\frac{1}{k}+O\left(\frac{1}{n}\right)
\end{aligned}
$$

(see Lemmas 2 and 3 in Akahira (2013)), it follows from (A.3) that

$$
E_{\theta, \gamma}\left(\hat{U} T_{(1)}\right)=\frac{1}{k \sqrt{\lambda_{2} n}}\left\{u(\gamma)-\lambda_{1}-\frac{\lambda_{3}}{2 \lambda_{2}}\right\}+O\left(\frac{1}{n \sqrt{n}}\right)
$$

hence (A.6) is obtained. Thus we complete the proof.

The proof of Theorem 3.1 By Taylor's expansion we have

$$
\begin{aligned}
& \hat{k}_{\theta}=k\left(\theta, X_{(1)}\right)=k\left(\theta, \gamma+\frac{T_{(1)}}{n}\right)=k(\theta, \gamma)+\frac{\partial k(\theta, \gamma)}{\partial \gamma} \cdot \frac{T_{(1)}}{n}+O_{p}\left(\frac{1}{n^{2}}\right) \\
& \frac{\partial \hat{k}_{\theta}}{\partial \gamma}=\frac{\partial k}{\partial \gamma}\left(\theta, X_{(1)}\right)=\frac{\partial k}{\partial \gamma}\left(\theta, \gamma+\frac{T_{(1)}}{n}\right)=\frac{\partial k(\theta, \gamma)}{\partial \gamma}+O_{p}\left(\frac{1}{n}\right) \\
& \hat{A}_{\theta}=A\left(\theta, X_{(1)}\right)=A\left(\theta, \gamma+\frac{T_{(1)}}{n}\right)=A(\theta, \gamma)+O_{p}\left(\frac{1}{n}\right) .
\end{aligned}
$$

Since by (A.8)

$$
\frac{1}{\hat{k}_{\theta}}=\frac{1}{k}\left\{1-\frac{1}{k}\left(\frac{\partial k}{\partial \gamma}\right) \frac{T_{(1)}}{n}+O_{p}\left(\frac{1}{n^{2}}\right)\right\}
$$

substituting (A.8), (A.9) and (A.10) for (3.2), we obtain from (2.6)

$$
\begin{aligned}
T_{(1)}^{*} & =n\left(X_{(1)}^{*}-\gamma\right)=n\left(X_{(1)}-\gamma\right)-\frac{1}{\hat{k}_{\theta}}-\frac{1}{\hat{k}_{\theta}^{3} n}\left(\frac{\partial \hat{k}_{\theta}}{\partial \gamma}\right)-\frac{1}{n} \hat{A}_{\theta} \\
& =T_{(1)}-\frac{1}{k}+\frac{1}{k^{2} n}\left(\frac{\partial k}{\partial \gamma}\right)\left(T_{(1)}-\frac{1}{k}\right)-\frac{1}{n} A+O_{p}\left(\frac{1}{n^{2}}\right)
\end{aligned}
$$




$$
\begin{aligned}
& =T_{(1)}-\frac{1}{k}-\frac{1}{n} A+\frac{1}{k n}\left(\frac{\partial}{\partial \gamma} \log k\right)\left(T_{(1)}-\frac{1}{k}\right)+O_{p}\left(\frac{1}{n^{2}}\right) \\
& =T_{(1)}-\frac{1}{k}+\frac{1}{k n}\left(\frac{\partial}{\partial \gamma} \log k\right) T_{(1)}+O_{p}\left(\frac{1}{n^{2}}\right),
\end{aligned}
$$

where $k=k(\theta, \gamma)$ and $A=A(\theta, \gamma)$. Hence we get (3.3). From (2.6), (3.3) and (A.2) it is easily seen that (3.4) holds, i.e. $E_{\gamma}\left(T_{(1)}^{*}\right)=O\left(1 / n^{2}\right)$. From (3.3), (A.2) and (A.3) we have

$$
\begin{aligned}
E_{\gamma}\left[T_{(1)}^{* 2}\right] & =\left\{1+\frac{2}{k^{2} n}\left(\frac{\partial k}{\partial \gamma}\right)\right\} E_{\gamma}\left[\left(T_{(1)}-\frac{1}{k}\right)^{2}\right]+O\left(\frac{1}{n^{2}}\right) \\
& =\left\{1+\frac{2}{k^{2} n}\left(\frac{\partial k}{\partial \gamma}\right)\right\}\left\{\frac{1}{k^{2}}-\frac{6\left(c_{\theta} b+a^{2} e^{\theta u}\right)}{a b k^{3} n}-\frac{2 A}{k n}\right\}+O\left(\frac{1}{n^{2}}\right) \\
& =\frac{1}{k^{2}}-\frac{6\left(c_{\theta} b+a^{2} e^{\theta u}\right)}{a b k^{3} n}-\frac{2 A}{k n}+\frac{2}{k^{3} n}\left(\frac{\partial}{\partial \gamma} \log k\right)+O\left(\frac{1}{n^{2}}\right),
\end{aligned}
$$

where $k=k(\theta, \gamma), a=a(\gamma), b=b(\theta, \gamma), c_{\theta}=c_{\theta}(\gamma), u=u(\gamma)$ and $A=A(\theta, \gamma)$. Since $k(\theta, \gamma)=$ $a(\gamma) e^{\theta u(\gamma)} / b(\theta, \gamma)$, it follows that

$$
\frac{a(\gamma) e^{\theta u(\gamma)}}{b(\theta, \gamma) k^{3}(\theta, \gamma)}=\frac{1}{k^{2}(\theta, \gamma)}
$$

hence by (A.11), (2.3) and (2.6)

$$
\begin{aligned}
E_{\gamma}\left[T_{(1)}^{*}{ }^{2}\right] & =\frac{1}{k^{2}}-\frac{6}{k^{2} n}\left(1+\frac{c_{\theta}}{a k}\right)-\frac{2}{k^{2} n}\left\{k A-\frac{1}{k}\left(\frac{\partial}{\partial \gamma} \log k\right)\right\}+O\left(\frac{1}{n^{2}}\right) \\
& =\frac{1}{k^{2}}-\frac{2}{k^{3} n}\left(\frac{\partial}{\partial \gamma} \log k\right)+O\left(\frac{1}{n^{2}}\right) .
\end{aligned}
$$

From (3.4) and (A.13) we get (3.5). Thus we complete the proof.

The proof of Theorem 4.1 By Taylor's expansion we have

$$
\begin{aligned}
& \hat{k}=k\left(\hat{\theta}_{M L}, X_{(1)}\right)=k\left(\hat{\theta}_{M L}, \gamma+\frac{T_{(1)}}{n}\right) \\
&=k(\theta, \gamma)+\left\{\frac{\partial k(\theta, \gamma)}{\partial \theta}\right\}\left(\hat{\theta}_{M L}-\theta\right)+\left\{\frac{\partial k(\theta, \gamma)}{\partial \gamma}\right\}\left(X_{(1)}-\gamma\right)+\frac{1}{2}\left\{\frac{\partial^{2} k(\theta, \gamma)}{\partial \theta^{2}}\right\}\left(\hat{\theta}_{M L}-\theta\right)^{2}+O_{p}\left(\frac{1}{n \sqrt{n}}\right) \\
&=k+\frac{1}{\sqrt{\lambda_{2} n}}\left(\frac{\partial k}{\partial \theta}\right) \hat{U}+\frac{1}{n}\left(\frac{\partial k}{\partial \gamma}\right) T_{(1)}+\frac{1}{2 \lambda_{2} n}\left(\frac{\partial^{2} k}{\partial \theta^{2}}\right) \hat{U}^{2}+O_{p}\left(\frac{1}{n \sqrt{n}}\right) .
\end{aligned}
$$


Since

$$
\begin{aligned}
& \frac{1}{\hat{k}}=\frac{1}{k}-\frac{1}{k^{2} \sqrt{\lambda_{2} n}}\left(\frac{\partial k}{\partial \theta}\right) \hat{U}-\frac{1}{k^{2} n}\left(\frac{\partial k}{\partial \gamma}\right) T_{(1)}-\frac{1}{2 k^{2} \lambda_{2} n}\left(\frac{\partial^{2} k}{\partial \theta^{2}}\right) \hat{U}^{2}+\frac{1}{k^{3} \lambda_{2} n}\left(\frac{\partial k}{\partial \theta}\right)^{2} \hat{U}^{2} \\
& +O_{p}\left(\frac{1}{n \sqrt{n}}\right), \\
& \hat{\lambda}_{j}=\lambda_{j}\left(\hat{\theta}_{M L}, X_{(1)}\right)=\lambda_{j}(\theta, \gamma)+O_{p}\left(\frac{1}{\sqrt{n}}\right) \quad(j=2,3), \\
& \frac{\partial^{j} \hat{k}}{\partial \theta^{j}}=\frac{\partial^{j} k}{\partial \theta^{j}}\left(\hat{\theta}_{M L}, X_{(1)}\right)=\frac{\partial^{j} k}{\partial \theta^{j}}(\theta, \gamma)+O_{p}\left(\frac{1}{\sqrt{n}}\right) \quad(j=1,2), \\
& \frac{\partial \hat{k}}{\partial \gamma}=\frac{\partial k}{\partial \gamma}\left(\hat{\theta}_{M L}, X_{(1)}\right)=\frac{\partial k}{\partial \gamma}(\theta, \gamma)+O_{p}\left(\frac{1}{\sqrt{n}}\right), \\
& \hat{A}=A\left(\hat{\theta}_{M L}, X_{(1)}\right)=A(\theta, \gamma)+O_{p}\left(\frac{1}{\sqrt{n}}\right),
\end{aligned}
$$

it follows from (4.4) that

$$
\begin{aligned}
T_{(1)}^{* *}= & n\left(X_{(1)}^{* *}-\gamma\right) \\
= & n\left(X_{(1)}-\gamma\right)-\frac{1}{\hat{k}}+\frac{1}{\hat{k}^{2} \hat{\lambda}_{2} n}\left(\frac{\partial \hat{k}}{\partial \theta}\right)\left\{\frac{1}{\hat{k}}\left(\frac{\partial \hat{\lambda}_{1}}{\partial \gamma}\right)+\frac{\hat{\lambda}_{3}}{2 \hat{\lambda}_{2}}\right\}-\frac{1}{\hat{k}^{3} n}\left(\frac{\partial \hat{k}}{\partial \gamma}\right) \\
& \quad-\frac{1}{2 \hat{k}^{2} \hat{\lambda}_{2} n}\left(\frac{\partial^{2} \hat{k}}{\partial \theta^{2}}\right)+\frac{1}{\hat{k}^{3} \hat{\lambda}_{2} n}\left(\frac{\partial \hat{k}}{\partial \theta}\right)^{2}-\frac{1}{n} \hat{A} \\
= & T_{(1)}-\frac{1}{k}-\frac{1}{n} A+\frac{1}{k^{2} \sqrt{\lambda_{2} n}}\left(\frac{\partial k}{\partial \theta}\right)\left\{\hat{U}+\frac{1}{\sqrt{\lambda_{2} n}}\left(\frac{1}{k}\left(\frac{\partial \lambda_{1}}{\partial \gamma}\right)+\frac{\lambda_{3}}{2 \lambda_{2}}\right)\right\} \\
& +\frac{1}{k^{2} n}\left(\frac{\partial k}{\partial \gamma}\right)\left(T_{(1)}-\frac{1}{k}\right)+\frac{1}{2 k^{2} \lambda_{2} n}\left\{\frac{\partial^{2} k}{\partial \theta^{2}}-\frac{2}{k}\left(\frac{\partial k}{\partial \theta}\right)^{2}\right\}\left(\hat{U}^{2}-1\right)+O_{p}\left(\frac{1}{n \sqrt{n}}\right),
\end{aligned}
$$

where $k=k(\theta, \gamma), A=A(\theta, \gamma)$ and $\lambda_{j}=\lambda_{j}(\theta, \gamma)(j=1,2,3)$, which derives (4.5) from (2.6). From (A.7), (A.14) and Lemmas A.1 and A.2 we obtain (4.6) and

$$
\begin{aligned}
V_{\theta, \gamma}\left(T_{(1)}^{* *}\right) & =E_{\theta, \gamma}\left[T_{(1)}^{* * 2}\right]+O\left(\frac{1}{n \sqrt{n}}\right) \\
& =E_{\theta, \gamma}\left[\left(T_{(1)}-\frac{1}{k}\right)^{2}\right]-\frac{2 A}{n} E_{\theta, \gamma}\left[T_{(1)}-\frac{1}{k}\right]+\frac{1}{k^{4} \lambda_{2} n}\left(\frac{\partial k}{\partial \theta}\right)^{2} E_{\theta, \gamma}\left(\hat{U}^{2}\right)
\end{aligned}
$$




$$
\begin{aligned}
& +\frac{2}{k^{2} \sqrt{\lambda_{2} n}}\left(\frac{\partial k}{\partial \theta}\right) E_{\theta, \gamma}\left[T_{(1)} \hat{U}-\frac{1}{k} \hat{U}+\frac{1}{\sqrt{\lambda_{2} n}}\left\{\frac{1}{k}\left(\frac{\partial \lambda_{1}}{\partial \gamma}\right)+\frac{\lambda_{3}}{2 \lambda_{2}}\right\}\left(T_{(1)}-\frac{1}{k}\right)\right] \\
& +\frac{2}{k^{2} n}\left(\frac{\partial k}{\partial \gamma}\right) E_{\theta, \gamma}\left[\left(T_{(1)}-\frac{1}{k}\right)^{2}\right]+\frac{1}{k^{2} \lambda_{2} n}\left(\frac{\partial^{2} k}{\partial \theta^{2}}\right) E_{\theta, \gamma}\left[\left(T_{(1)}-\frac{1}{k}\right)\left(\hat{U}^{2}-1\right)\right] \\
& -\frac{2}{k^{3} \lambda_{2} n}\left(\frac{\partial k}{\partial \theta}\right)^{2} E_{\theta, \gamma}\left[\left(T_{(1)}-\frac{1}{k}\right)\left(\hat{U}^{2}-1\right)\right]+O\left(\frac{1}{n \sqrt{n}}\right) \\
& =\frac{1}{k^{2}}-\frac{6\left(c_{\theta} b+a^{2} e^{\theta u}\right)}{a b k^{3} n}-\frac{2 A}{k n}+\frac{1}{k^{4} \lambda_{2} n}\left(\frac{\partial k}{\partial \theta}\right)^{2}+\frac{2}{k^{4} n}\left(\frac{\partial k}{\partial \gamma}\right)+O\left(\frac{1}{n \sqrt{n}}\right) .
\end{aligned}
$$

Since

$$
\frac{\partial}{\partial \theta} \log k(\theta, \gamma)=u(\gamma)-\lambda_{1}(\theta, \gamma)
$$

it follows from (2.6), (A.12) and (A.15) that

$$
\begin{aligned}
V_{\theta, \gamma}\left(T_{(1)}^{* *}\right)=\frac{1}{k^{2}} & -\frac{6}{k^{2} n}\left(1+\frac{c_{\theta}}{a k}\right)-\frac{2}{k^{2} n}\left\{k A-\frac{1}{k}\left(\frac{\partial}{\partial \gamma} \log k\right)\right\} \\
& +\frac{1}{k^{2} \lambda_{2} n}\left(u(\gamma)-\lambda_{1}\right)^{2}+O\left(\frac{1}{n \sqrt{n}}\right) \\
=\frac{1}{k^{2}} & -\frac{2}{k^{3} n}\left(\frac{\partial}{\partial \gamma} \log k\right)+\frac{1}{k^{2} \lambda_{2} n}\left(u(\gamma)-\lambda_{1}\right)^{2}+O\left(\frac{1}{n \sqrt{n}}\right),
\end{aligned}
$$

where $a=a(\gamma)$ and $c_{\theta}=c_{\theta}(\gamma)=a^{\prime}(\gamma)+\theta a(\gamma) u^{\prime}(\gamma)$, which shows that (4.7) holds. Thus we complete the proof.

\section{Acknowledgements}

The authors thank the referee for kind comments. Research was supported in part by Grant-inAid for Scientific Research (B), No.23340022, JSPS.

\section{References}

[1] Akahira, M. (1981). On asymptotic deficiency of estimators. Australian Journal of Statistics, $23,67-72$.

[2] Akahira, M. (1986). The Structure of Asymptotic Deficiency of Estimators. Queen's Papers in Pure and Applied Mathematics, 75, Queen's University Press, Kingston, Canada. 
[3] Akahira, M. (2013). Second order asymptotic comparison of the MLE and MCLE of a natural parameter for a truncated exponential family of distributions. Mathematical Research Note 2013-001, Institute of Mathematics, University of Tsukuba. To appear in Annals of the Institute of Statistical Mathematics. Published online: 21 February 2015.

[4] Akahira, M. and Ohyauchi, N. (2015). Second order asymptotic loss of the MLE of a truncation parameter for a two-sided truncated exponential family of distributions. Mathematical Research Note 2015-002, Institute of Mathematics, University of Tsukuba.

[5] Akahira, M., Hashimoto, S., Koike, K. and Ohyauchi, N. (2014). Second order asymptotic comparison of the MLE and MCLE for a two-sided truncated exponential family of distributions. Mathematical Research Note 2014-001, Institute of Mathematics, University of Tsukuba. To appear in Communications in Statistics -Theory and Methods.

[6] Bar-Lev, S. K. (1984). Large sample properties of the MLE and MCLE for the natural parameter of a truncated exponential family. Annals of the Institute of Statistical Mathematics, 36, Part A, 217-222.

[7] Basu, D. (1977). On the elimination of nuisance parameters. Journal of the American Statistical Association, 72, 355-366.

[8] Fisher, R. A. (1925). Theory of statistical estimation. Proceedings of Cambridge Philsophical Society, 22, 700-725.

[9] Hodges, J. L. and Lehmann, E. L. (1970). Deficiency. Annals of Mathematical Statistics, 41, 783-801.

[10] Rao, C. R. (1961). Asymptotic efficiency and limiting information. Proceedings of the Third Berkeley Symposium on Mathematical Statistics and Probability, Vol. 1, 531-545.

[11] Voinov, V. G. and Nikulin, M. S. (1993). Unbiased Estimaters and Their Applications, Vol. 1: Univariate Case. Dordrecht: Kluwer Academic Publishers. 